\title{
PREVALÊNCIA DE INFARTOS RENAIS EM NECROPSIAS DE CHAGÁSICOS CRÔNICOS
}

\author{
Soraya Vecci Mohallem, Sidney Gonçalves Ramos, \\ Marlene Antônia dos Reis, Daniel Damião Gomes Seabra \\ e Vicente de Paula Antunes Teixeira
}

\begin{abstract}
O infarto renal (IR) é usualmente secundârio a obstrução arterial por êmbolos originários do coraçâo. O chagásico crônico pode apresentar alterações cardíacas que originam trombos intracaitútarios mesmo sem insuficiência cardiaca congestiva (ICC). Neste trabalho avaliou-se comparativamente, a freqüencia de IR em chagásicos crônicos, nas diferentes formas anátomo-clinicas e em não chagásicos. Realizou-se a revisâo dos laudos de necropsias de indîtiduos com idade maior ou igual a 20 anos. Em 259 necropsias, 78 (30,1\%) eram de chagâsicos crôniicos, destes 19 (24,4\%) desenvolveram IR, enquanto $27(15,0 \%)$ não chagásicos apresentaram IR. A idade dos chagásicos com IR foi semelbante à dos näo chagásicos. Encontrou-se predominio significante de IR e trombose nos chagásicos. Observou-se uma prevalência significantemente maior de IR nos chagâsicos com ICC $(52,6 \%)$ quando comparados às outras formas anátomo-clinicas da doença e aos não chagásicos. Concluiu-se que o IR foi mais freqüente nos chagásicos, especialmente naqueles que desenvolveram ICC, provavelmente colaborando nas eventuais manifestações renais e alterações hemodinâmicas sistêmicas nestes pacientes.
\end{abstract}

Palauras-chanes: Infarto renal. Doença de Chagas. Rim. Trypanosoma cruzi.

O infarto renal (IR) branco ou isquêmico ocorre como consequêencia da obstrução dos vasos renais, sendo usualmente secundário a obstrução arterial, mais comumente de origem embólica ${ }^{16}$. Estes êmbolos freqüentemente têm origem no coraçâo ${ }^{68}$. Entretanto, a embolização renal isolada mais freqüente é a ateromatosa, resultado da arteriosclerose aórtica ${ }^{11}$. Os rins são sedes frequientes de infartos, pois recebem um quarto do débito cardíaco. Por outro lado, o coração é fonte de êmbolo sistêmico em mais de $94 \%$ dos pacientes. Portanto, pacientes com cardiopatias que predipõem à formação de trombos intracavitários são potenciais vítimas do IR embólico. Destacamse as doenças valvares cardíacas, a fibrilação atrial e o infarto agudo do miocárdio como formadores de trombos murais".

O paciente chagásico crônico freqüentemente apresenta alterações do ritmo cardíaco, sendo a extra-sístole a arritmia predominante. Os fenômenos embólicos, a partir das tromboses

Disciplina de Patologia Geral da Faculdade de Medicina do Triângulo Minciro, Uberaba, MG.

Endereço para correspondência: Profa. Mariene Antônia dos Reis. Disc. Patologia Geral/FMTM. Pça Manoel Terra s/n, 38015-050 Uberaba, MG.

Recebido para publicação em 23/11/95. murais do endocárdio, na pequena e na grande circulação, podem ocorrer mesmo na ausência de insuficiência cardiaca e gerar infartos em vários órgãos, porém a literatura é escassa em relação ao IR e a doença de Chagas $^{913}$.

O objetivo deste trabalho é de avaliar, comparativamente, a freqüência de IR em chagásicos crônicos, nas suas diferentes formas anátomo-clínicas e em não chagásicos.

\section{MATERIAL E MÉTODOS}

O material foi proveniente de necropsias completas, realizadas no Hospital Escola da Faculdade de Medicina do Triângulo Mineiro, Uberaba (MG). Foram selecionados, através da leitura dos laudos de necropsias, no período de 1984 a 1994, indivíduos com idade maior ou igual a 20 anos, portadores da doença de Chagas crônica e indivíduos não chagásicos. Foram considerados chagásicos crônicos, os indivíduos que apresentavam as reaçôes de Guerreiro-Machado, imunofluorescência indireta e de hemaglutinação positivas para Trypanosoma cruzi realizadas no líquido pericárdico colhido à necropsia. Nos não chagásicos estas reações foram negativas. Os chagásicos foram classificados segundo a forma anátomo-clínica, a saber: assintomáticos 
Mohallem SV, Ramos SG, Reis MA. Seabra DDG. Teixeira VPA. Prevalência de infartos renais em necropsias de chagásicos crônicos. Revista da Sociedade Brasileira de Medicina Tropical 29:571-574, nov-dez 1996.

(forma indeterminada), os falecidos abruptamente sem manifestações prévias da doença (morte súbita), aqueles que faleceram na vigência de insuficiência cardíaca congestiva (ICC) e os portadores de enteromegalias (mega, esôfago e/ou cólon). Seguiu-se um protocolo colhendo os seguintes dados: 1) a idade, a cor e o sexo; 2) presença de IR, branco (isquêmico); 3) presença de arteriosclerose na aorta e/ou nas artérias renais; 4) presença de trombose nestes vasos e/ou intracardíaca.

A análise estatística foi feita através dos testes " $\chi$ " e "t" de Student. As diferenças observadas foram consideradas estatisticamente significantes quando a probabilidade de rejeiçâo da hipótese de nulidade foi menor que $5 \%(p<0,05)$.

\section{RESULTADOS}

Dos 259 laudos de necropsias, 78 referiamse a chagásicos; destes, $19(24,4 \%)$ apresentavam IR. Dos 181 não chagásicos, $27(14,9 \%)$ apresentavam IR. A Tabela 1 mostra a distribuição do IR comparativamente entre

Tabela 1 - Distribuchào dos infartos renais em cbagúsicos crônicos e não chagásicos necropsiados.

\begin{tabular}{|c|c|c|c|c|}
\hline & \multicolumn{2}{|c|}{ Chagásicos } & \multicolumn{2}{|c|}{ Não chagásicos } \\
\hline & $n^{2}$ & $\%$ & $n$ & $\%$ \\
\hline Infarto tenal & 19) & 24,4 & 27 & 14.9 \\
\hline Sem infarto renal & 59 & 75.6 & 154 & 85.1 \\
\hline Total & 78 & 100 & 181 & 100 \\
\hline
\end{tabular}

$\overline{\chi^{2}-3.33 ; \bar{p}}=0,0681$.

chagásicos e não chagásicos. A Tabela 2 exibe a distribuição do IR nos chagásicos e não chagásicos com relação ao sexo, a cor, a idade, a presença de arteriosclerose na aorta e/ou nas artérias renais, a trombose nestes vasos e/ou

Tabela 2 - Distribuição dos infartos renais nos cbagásicos crônicos $e$ não chagásicos necropsiacios. com relaça ao sexo, a cor. a $e$ nat chagasicos necropsiacios. com relacáo
idade, a presença de arteriosclerose e trombose.

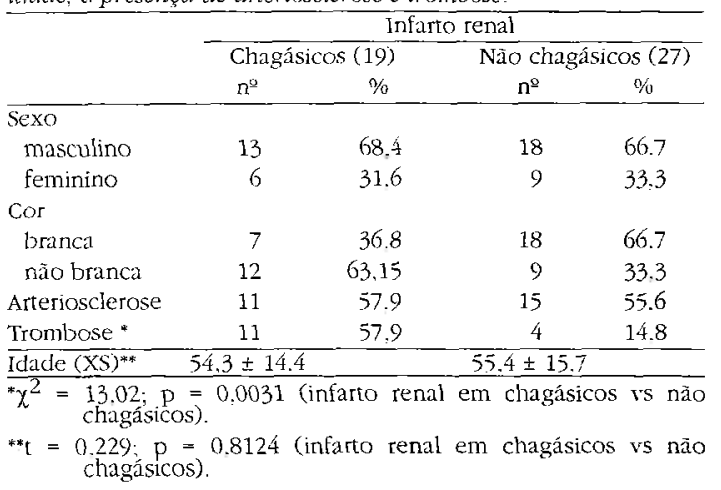

intracardíaca. Não foram observadas diferenças estatisticamente significantes quando se çomparou o sexo, a cor, a idade e a presença de arteriosclerose entre o grupo chagásico e o não chagásico. A Tabela 3 mostra a prevalência de IR nos indivíduos chagásicos crônicos, nas suas diferentes formas anátomoclínicas.

Tabela 3 - Distribuiçâo da freqüêucia dos infartos renais nos individuos chagásicos crônicos nas suas diferentes formas anátomo-clinicas e en nấo chagásicos necropsiacios.

\begin{tabular}{|c|c|c|c|c|c|c|}
\hline & \multicolumn{2}{|c|}{ Com infarto } & \multicolumn{2}{|c|}{ Sem infarto } & \multicolumn{2}{|c|}{ Total } \\
\hline & $\mathrm{n}^{\underline{o}}$ & $\%$ & $\mathrm{n}^{\mathrm{o}}$ & $\%$ & $\mathrm{n}^{\mathrm{Q}}$ & $\%$ \\
\hline \multicolumn{7}{|l|}{ Chagásicos } \\
\hline indeterminada & 4 & 18.2 & 18 & 81.8 & 22 & 100 \\
\hline mega & 4 & 13,3 & 26 & 86.7 & 30 & 100 \\
\hline morte súbita & 1 & 33.3 & 2 & 66.7 & 3 & 100 \\
\hline $\mathrm{ICC}^{*}$ & 10 & 43.5 & 13 & 56.5 & 23 & 100 \\
\hline Nào chagásicos & 27 & 14.9 & 154 & 85.1 & 181 & 100 \\
\hline
\end{tabular}

*ICC vs outras formas anátomo-clínicas; $\chi^{2}=5,08: p=0,024$ "ICC vs não chagásicos: $\chi^{2}=9,37 ; \mathrm{p}=0,0022$.

\section{DISCUSSÃO}

No presente estudo, dos 259 indivíduos estudados, encontrou-se IR em $17,8 \%$, porcentagem maior que a relatada em outros estudos em necropsias, variando de $0,48 \%$ a $5,4 \%$ (11 $^{12}$. Em relação a cor, nos chagásicos crônicos o IR foi mais prevalente nos "não brancos" $(63,2 \%)$. Peterson e McDonald ${ }^{11}$ estudando 113 casos de IR encontraram somente cinco indivíduos "não brancos". Talvez este dado esteja relacionado a fatores socioeconômicos e às características das populaçôes dos locais onde foram realizadas as pesquisas, porém devem ser melhor investigados. Observou-se que os IR foram mais freqüentes em indivíduos do sexo masculino, tanto nos chagásicos crônicos $(68,4 \%)$ quanto nos não chagásicos $(66,7 \%)$. Alguns autores encontraram uma maior prevalência de IR em indivíduos do sexo masculino $(67,3 \%)^{15}$, no entanto outros trabalhos mostraram não haver predominância entre os $\operatorname{sexos}^{613}$, neste último estudo foram excluidos os casos de êmbolos ateromatosos que predominaram no sexo masculino.

Como se observa nas Tabelas 1 e 3, o IR foi mais freqüente nos chagásicos crônicos $(24,4 \%)$, sendo predominante nos indivíduos com ICC (43,5\%). Alguns autores encontraram doença cardíaca em $75 \%$ a $95 \%$ dos casos com $\mathrm{IR}^{2}{ }^{12}$. No presente estudo observou-se ainda um predominio significante de IR acompanhado de trombose nos chagásicos crônicos. A embolia 
Mohallem SV, Ramos SG, Reis MA, Seabra DDG, Teixeira VPA. Pretalência de infartos renais em necropsias de chagásicos crônicos. Retista da Sociedade Brasileira de Medicina Tropical 29:571-574, nou-dez 1996.

é a principal causa de IR, quando se compara a etiologia do IR e as imagens obtidas através da tomografia computadorizada ${ }^{-}$, sendo $O$ coração fonte de êmbolo sistêmico em mais de 90\% dos pacientes ${ }^{4}$. Estes êmbolos geralmente provem de trombos murais de pacientes com acometimento cardíaco severo ${ }^{10}$. Portanto, pacientes com cardiopatias que predispóem à formação de trombos intracavitários são potencias vítimas do IR embólico". Entre estas, destacam-se as doenças valvares cardíacas-a a fibrilação atrial ${ }^{1617}$ e o infarto agudo do miocardio ${ }^{-}$. Aproximadamente $23 \%$ dos pacientes com endocardite infecciosa desenvolvem $\mathrm{IR}^{\circ}$. Entretanto, em apenas um trabalho encontrouse IR exclusivamente relacionado a doença de Chagas $^{13}$.

A doença de Chagas tem o coração como um dos órgãos alvos. O processo inflamatório no miocárdio, os distúrbios de condução, a lesão vorticilar esquerda e a própria dilatação das câmaras cardíacas, são fatores que predispõem a formação de trombos intracavitários ${ }^{13}$. Estes trombos são freqüentemente fontes de êmbolos que apresentam grande chance de alcançarem a circulaçâo renal, pois esta recebe cerca de $25 \%$ do débito cardíaco. O chagásico com ICC apresenta mais freqüentemente estas manifestações cardíacas, contribuindo para a formaçâo e o desprendimento de trombos intracavitários que são encontrados em $75 \%$ destes pacientes ${ }^{1}$. A propósito, Duggan ${ }^{2}$ descreveu um caso de ICC gerando IR.

Em ambos os grupos do presente trabalho encontrou-se arteriosclerose na aorta e/ou artérias renais associada ao IR. Este fato colaboraria para explicar a presença de IR entre os não chagásicos e nos chagásicos sem ICC. Alguns autores mostraram que a causa de embolização renal isolada mais freqüente é a ateromatosa ${ }^{11}$. Em pacientes com arteriosclerose severa da aorta, a incidência de embolização ateromatosa tem variado de $3 \% 0^{3}$ a $16 \%$ e em pacientes com aneurisma de aorta tem variado de $7 \%$ a $31 \%{ }^{14}$. O rim é um dos orgãos mais comumente afetados, especialmente pela oclusão arterial ateromatosa em pequenas e médias artérias renais ${ }^{318}$.

Portanto, nos indivíduos chagásicos crônicos houve predomínio de IR, sendo mais freqüente nos portadores de ICC. Apesar do IR ter pequena repercussão clínica, dependendo da extensão acometida pode contribuir com as alterações hemodinâmicas. Em um paciente que se encontra já bastante comprometido sistemicamente, como os chagásicos crônicos, justificam-se a realização de mais estudos sobre as lesões renais e suas conseqüências para maior entendimento desta doença $e$ abordagem clínica do paciente.

\section{SUMMARY}

Renal infarction (RI) is usually secondary to arterial obstruction due to emboli originating from the beart. Cbronic chagasic patients may present cardiac alterations originating from intracavitary thrombi, even uithout congestive beart failure (CHF). In this study RI incidence was comparatively evaluated in cbronic chagasic individuals, in different anatomoclinic forms and in non chagasic individuals. There bas been a review on necropsy reports of inditiduals aged 20 or over. In 259 necropsies, $78(30.1 \%)$ were chagasics, and 19 of them (24.4\%) developed RI, while 27 (15.0\%) of the non chagasic indiuiduals presented RI. The ages of chagasics with RI were similar to those of non chagasic indiniduals. A significant prevalence of $R I$ and thrombosis among chronic cbagasic individuals bas been found. A significantly bigher prevalence of RI among chronic chagasics baving CHF $(52.6 \%)$ was observed when they were compared to other forms of chronic Chagas disease and when compared to non chagasic individuals. It was concluded that RI was more frequent in chronic chagasic individuals, specially those who developed CHF, which probably played a role in the renal manifestations and systemic bemodynamic changes in those patients.

Key-words: Renal infarction. Chagas disease. Kidney. Trypanosoma cruzi.

\section{AGRADECIMENTOS}

Aos funcionários da Disciplina de Patologia Geral (FMTM). À Fundação de Ensino e Pesquisa de Uberaba - FUNEPU, à Fundação de Amparo à Pesquisa do Estado de Minas Gerais - FAPEMIG e ao Conselho Nacional de Desenvolvimento Científico e Tecnológico - CNPq.

\section{REFERÊNCIAS BIBLIOGRÁFICAS}

1. Cotran RS, Kumar VY, Robbins SL. Rim. In: Cotran RS, Kumar VY, Robbins SL (eds) Robins Patologia Estrutural e Funcional, 4a edição, Ed Guanabara Koogan, Rio de Janeiro, p.833-891, 1991.

2. Duggan ML. Acute Renal Infarction. The Journal of Urology 90:669-676, 1963. 
Mohallem SV, Ramos SG, Reis MA, Seabra DDG, Teixeira VPA. Prevalência de infartos renais em necropsias de chagásicos crônicos. Revista da Sociedade Brasileira de Medicina Tropical 29:571-574, nov-dez 1996.

3. Flory CM. Arterial occlusions produced by emboli from eroded aortic atheromatous plaques. The American Journal of Pathology 21:549-565, 1945.

4. Goldberg G. Renal infarction. Annals of Emergency Medicine 14:611-614, 1985.

5. Greendyke RM, Akamatsu Y. Atheromatous embolism as a cause of renal failure. The Journal of Urology 83:231-237, 1960.

6. Hoxie HJ, Coggin CB. Renal infarction. Statistical study of two hundred and five cases and detailed report of an unusual case. Archives of Internal Medicine 65:587-594, 1940.

7. Kim SH, Park JH, Han JK, Han MC, Kim S, Lee JS. Infarction of the kidney: role of contrast enhanced magnetic resonance imaging (MRI). Journal of Computer Assisted Tomography 16:924-928, 1992.

8. Lessman RK, Johnson SF, Coburn JW, Kaufman JJ. Renal artery embolism: clinical features and longterm follow-up of 17 cases. Annals of Internal Medicine 89:477-482, 1978.

9. Lopes ER, Chapadeiro E, Tafuri W, Prata AR. Patologia das principais doenças tropicais no Brasil. In: Brasileiro Filho G, Pittella JEH, Pereira FEL, Bambirra EA, Barbosa AJA (eds) Bogliolo Patologia, 5a edição, Ed Guanabara Koogan SA, Rio de Janeiro, p.1103-1201, 1994.

10. McRae CU. Acute renal artery occlusion. New Zealand Medical Journal 82:344-346, 1975.
11. Peterson NE, MCDonald DF. Renal embolization. The Journal of Urology 100:140-145, 1968.

12. Regan FC, Crabtree EG. Renal Infarction: a clinical and possible surgical entity. The Journal of Urology 59:981-1018, 1948.

13. Teixeira VPA, Brandão MC, Almeida HO. Embolia arterial múltipla por trombose atrial esquerda em chagásico sem insuficiência cardíaca. Revista Goiana de Medicina 32:179-183, 1986.

14. Thurbeckwm, Castleman B. Atheromatous emboli to the kidneys after aortic surgery. New England Journal of Medicine 257:442-447, 1957.

15. Tse RL, Leberman PR. Acute renal occlusion: etiology, diagnosis and treatment. Report of a case with subsequent revascularization. The Jornal of Urology 108:32-34, 1972.

16. Weiss MA, Pollak VE. Renal cortical necrosis, infarction, and atheroembolic disease. In: Tischer CC, Brenner BM (eds) JB: Renal Pathology with clinical and functional correlations, JB Lippincott Company, Philadelphia, p.700-727, 1989.

17. Wong WS, Moss AA, Ferdele MP, Cochran ST, London SS. Renal infatction: CT diagnosis and correlation between $\mathrm{CT}$ findings and etiologies. Radiology 150:201-205, 1984.

18. Zak PG, Elias K: Embolization with material from atheromata. American Journal of Medicine 218:510-515, 1949. 